\title{
The Amati relation in the "fireshell" model
}

\author{
R. Guida ${ }^{1,2}$, M. G. Bernardini ${ }^{1,2}$, C. L. Bianco ${ }^{1,2}$, L. Caito ${ }^{1,2}$, M. G. Dainotti ${ }^{1,2}$, and R. Ruffini ${ }^{1,2,3}$ \\ 1 ICRANet and ICRA, Piazzale della Repubblica 10, 65122 Pescara, Italy \\ 2 Dipartimento di Fisica, Università di Roma "La Sapienza", Piazzale Aldo Moro 5, 00185 Roma, Italy \\ e-mail: [roberto.guida;maria.bernardini;bianco;letizia.caito;dainotti;ruffini]@icra.it \\ 3 ICRANet, Université de Nice Sophia Antipolis, Grand Château, BP 2135, 28 avenue de Valrose, 06103 Nice Cedex 2, France
}

Received 6 June 2008 / Accepted 23 June 2008

\section{ABSTRACT}

\begin{abstract}
Context. The cosmological origin of gamma-ray bursts (GRBs) has been firmly established, with redshifts up to $z=6.29$. They are possible candidates for use as "distance indicators" for testing cosmological models in a redshift range hardly achievable by other cosmological probes. Asserting the validity of the empirical relations among GRB observables is now crucial for their calibration. Aims. Motivated by the relation proposed by Amati and collaborators, we look within the "fireshell" model for a relation between the peak energy $E_{\mathrm{p}}$ of the $v F_{v}$ total time-integrated spectrum of the afterglow and the total energy of the afterglow $E_{\text {aft }}$, which in our model encompasses and extends the prompt emission.

Methods. The fit within the fireshell model, as for the "canonical" GRB 050315, uses the complete arrival time coverage given by the Swift satellite. It is performed simultaneously, self-consistently, and recursively in the four BAT energy bands (15-25 keV, $25-50 \mathrm{keV}, 50-100 \mathrm{keV}$, and 100-150 keV), as well as in the XRT one (0.2-10 keV). It uniquely determines the two free parameters characterizing the GRB source, the total energy $E_{\text {tot }}^{\mathrm{e}^{ \pm}}$of the $\mathrm{e}^{ \pm}$plasma and its baryon loading $B$, as well as the effective CircumBurst Medium (CBM) distribution. We can then build two sets of "gedanken" GRBs varying the total energy of the electron-positron plasma $E_{\text {tot }}^{\mathrm{e}^{ \pm}}$and keeping the same baryon loading $B$ of GRB 050315. The first set assumes the one obtained in the fit of GRB 050315 for the effective CBM density. The second set assumes instead a constant CBM density equal to the average value of the GRB 050315 prompt phase.

Results. For the first set of "gedanken" GRBs we find a relation $E_{\mathrm{p}} \propto\left(E_{\mathrm{aft}}\right)^{a}$, with $a=0.45 \pm 0.01$, whose slope strictly agrees with the Amati one. Such a relation, in the limit $B \rightarrow 10^{-2}$, coincides with the Amati one. Instead, no correlation is found in the second set of "gedanken" GRBs.

Conclusions. Our analysis excludes the proper GRB (P-GRB) from the prompt emission, extends all the way to the latest afterglow phases, and is independent of the assumed cosmological model, since all "gedanken" GRBs are at the same redshift. The Amati relation, on the other hand, includes the P-GRB, focuses only on the prompt emission, being therefore influenced by the instrumental threshold that fixes the end of the prompt emission, and depends on the assumed cosmology. This might explain the intrinsic scatter observed in the Amati relation.
\end{abstract}

Key words. gamma rays: bursts - gamma rays: observations - black hole physics - ISM: structure - distance scale

\section{Introduction}

The detection of gamma-ray bursts (GRBs) up to very high redshifts (up to $z=6.29$, see Tagliaferri et al. 2005), their high observed rate of one every few days, and the progress in the theoretical understanding of these sources all make them useful as cosmological tools, complementary to supernovae Ia, which are observed only up to $z=1.7$ (Leibundgut 2001; Riess et al. 2001). One of the hottest topics on GRBs is the possible existence of empirical relations between GRB observables (Amati et al. 2002; Ghirlanda et al. 2004; Yonetoku et al. 2004; Liang \& Zhang 2005; Firmani et al. 2006; Amati et al. 2008), which may lead, if confirmed, to using GRBs as tracers of models of universe. The first empirical relation, discovered when analyzing the BeppoSAX so-called "long" bursts with known redshift, was the "Amati relation" (Amati et al. 2002). It was found that the isotropic-equivalent radiated energy of the prompt emission $E_{\text {iso }}$ is correlated with the cosmological rest-frame $v F_{v}$ spectrum peak energy $E_{\mathrm{p}, \mathrm{i}}: E_{\mathrm{p}, \mathrm{i}} \propto\left(E_{\mathrm{iso}}\right)^{a}$, with $a=0.52 \pm 0.06$ (Amati et al. 2002). The existence of the Amati relation has been confirmed by studying a sample of GRBs discovered by Swift, with $a=0.49_{-0.05}^{+0.06}$ (Sakamoto et al. 2006; Amati 2006).

Swift has for the first time made it possible to obtain high quality data in selected energy bands from the GRB trigger time all the way to the latest afterglow phases (Gehrels et al. 2004). This has given us the opportunity to apply our theoretical "fireshell" model, thereby obtaining detailed values for its two free parameters, namely for the total energy $E_{\text {tot }}^{\mathrm{e}^{ \pm}}$and the baryon loading $B$ of the fireshell, as well as for the effective density and filamentary structure of the CircumBurst Medium (CBM). From this we were able to compute multi-band light curves and spectra, both instantaneous and time-integrated, compared with selected GRB sources, such as GRB 050315.

In the "fireshell" model, $E_{\text {tot }}^{\mathrm{e}^{ \pm}}$comprises two different components: (i) the proper GRB (P-GRB) with energy $E_{\mathrm{P}-\mathrm{GRB}}$, emitted at the moment when the $\mathrm{e}^{+} \mathrm{e}^{-}$-driven accelerating baryonic matter reaches transparency, and (ii) the following afterglow phase with energy $E_{\text {aft }}$, with the decelerating baryons interacting with the CBM (Ruffini et al. 2001). These two phases are clearly 
distinguishable by their relative intensity and temporal separation in arrival time. We have

$E_{\text {tot }}^{\mathrm{e}^{ \pm}}=E_{\mathrm{P}-\mathrm{GRB}}+E_{\mathrm{aft}}$.

What is usually called the "prompt emission" corresponds within the fireshell model to the P-GRB together with the peak of the afterglow (see below, e.g. Ruffini et al. 2001, 2006, 2007; Dainotti et al. 2007; Bernardini et al. 2007; Caito et al. 2008; Bianco et al. 2008, and references therein).

Among the crucial issues raised by the Amati relation, there are its theoretical explanation and its possible dependence on the assumed cosmological model. We examined a set of "gedanken" GRBs, all at the same cosmological redshift of GRB 050315. Such a set assumes the same fireshell baryon loading and effective CBM distribution as GRB 050315 and each "gedanken" GRB differs from the others uniquely by the value of its total energy $E_{\text {tot }}^{\mathrm{e}^{ \pm}}$. We then considered a second set of "gedanken" GRBs, differing from the previous one by assuming a constant effective CBM density instead of the one inferred for GRB 050315. In both these sets, we looked for a relation between the isotropicequivalent radiated energy of the entire afterglow $E_{\text {aft }}$ and the corresponding time-integrated $v F_{v}$ spectrum peak energy $E_{\mathrm{p}}$ :

$E_{\mathrm{p}} \propto\left(E_{\mathrm{aft}}\right)^{a}$.

In Sect. 2 we recall the main features of the "fireshell" model, and in Sect. 3 the main features of the GRB 050315 fitting procedure. In Sect. 4 we then present the derivation of the $E_{\mathrm{p}}-E_{\text {aft }}$ relation for two sets of "gedanken" GRBs. The results of this analysis and their discussion are shown in Sect. 5. In Sect. 6 we present the conclusions, strongly confirming the validity of the Amati relation.

\section{The "fireshell" model and the canonical GRB scenario}

Our "fireshell" model assumes that all GRBs originate from the gravitational collapse to a black hole (Ruffini et al. 2001, 2007). The $\mathrm{e}^{ \pm}$plasma created in the process of the black hole formation expands as a spherically symmetric "fireshell" with a constant width of $\sim 10^{8} \mathrm{~cm}$ in the laboratory frame, i.e. the frame in which the black hole is at rest. We have only two free parameters characterizing the source, the total energy $E_{\text {tot }}^{\mathrm{e}^{ \pm}}$of the $\mathrm{e}^{ \pm}$plasma and its baryon loading $B \equiv M_{\mathrm{B}} c^{2} / E_{\mathrm{tot}}^{\mathrm{e}^{ \pm}}$, where $M_{\mathrm{B}}$ is the total baryons' mass (Ruffini et al. 2000). They fully determine the optically thick acceleration phase of the fireshell, which lasts until the transparency condition is reached and the P-GRB is emitted (Ruffini et al. 2001). We recall that the P-GRB is sometimes considered a "precursor" of the main GRB event in the current literature.

After this optically thick acceleration phase, the optically thin deceleration phase starts, with the afterglow emission due to the collision between the remaining accelerated baryonic matter and the CBM. This emission clearly depends on the parameters describing the effective CBM distribution: its density $n_{\mathrm{cbm}}$ and the ratio $\mathcal{R} \equiv A_{\text {eff }} / A_{\text {vis }}$ between the effective emitting area of the fireshell $A_{\text {eff }}$ and its total visible area $A_{\text {vis }}$ (Ruffini et al. 2002, 2004, 2005a). The radiation emitted during the afterglow is assumed to have a thermal spectrum in the co-moving frame of the fireshell. Due to the temporal evolution of the fireshell temperature and to the Doppler effect implied by its ultra-relativistic expansion, the observed afterglow spectra are non-thermal because they are convolutions of thousands of thermal spectra with different temperatures over the corresponding EQuiTemporal
Surfaces (EQTSs, the surfaces of equal arrival time of the photons at the detector, see Bianco \& Ruffini 2004, 2005) and over the observation time (Ruffini et al. 2004; Bernardini et al. 2005).

We indeed define within our model the "canonical GRB" light curve as made by two sharply physical different components: the P-GRB and the afterglow (Ruffini et al. 2001, 2007; Bianco et al. 2008). The former has the imprint of the black hole formation, a harder spectrum, and no spectral lag (Bianco et al. 2001; Ruffini et al. 2005b). The latter presents a clear hard-to-soft spectral evolution in time, and it consists of three well-defined different regimes: a rising branch, a peak, and a decaying tail (Ruffini et al. 2001). The ratio between the total timeintegrated luminosities (i.e. the total energies) of the P-GRB and the afterglow, as well as their arrival time separation, are ruled by $E_{\text {tot }}^{\mathrm{e}^{ \pm}}$and $B$ (Ruffini et al. 2000, 2001). When $B \lesssim 10^{-5}$, the $\mathrm{P}-\mathrm{GRB}$ is the leading contributor to the emission and the afterglow is negligible: we have a "genuine" short GRB (Ruffini et al. 2001; Bernardini et al. 2007; Bianco et al. 2008). When $10^{-4} \lesssim B \lesssim 10^{-2}$, instead, the afterglow contribution is generally predominant (for the existence of the upper limit $B \lesssim 10^{-2}$ see Ruffini et al. 2000). Still, this last case presents two distinct possibilities: the afterglow peak luminosity can be either higher or lower than the P-GRB one. This last case, i.e. an afterglow with the total time-integrated luminosity higher than the P-GRB one but with a lower peak luminosity, is indeed explicable in terms of a peculiarly low average value of the CBM density $\left(n_{\mathrm{cbm}} \sim 10^{-3}\right.$ particles $\left./ \mathrm{cm}^{3}\right)$, compatible with a galactic halo environment. Such a low average CBM density "deflates" the afterglow peak luminosity, which therefore has to last longer since its total energy is fixed by the value of $B$. This is the class of the "fake" short GRBs, which present an initial spikelike emission followed by a prolonged soft bump (see Bernardini et al. 2007, 2008; Bianco et al. 2008, and references therein) and includes the sources analyzed by Norris \& Bonnell (2006).

What is usually called the "prompt emission" neglects all the above analysis; in fact, it comprises the P-GRB and the first two regimes of the afterglow, namely the rising branch and the peak. What is usually called the "afterglow" is just the third regime, its decaying tail (see e.g. Bernardini et al. 2005; Ruffini et al. 2006, 2007; Dainotti et al. 2007; Bernardini et al. 2007; Bianco et al. 2008). Within our approach there is no way to find a separation between the end of the "prompt emission" and the beginning of the decaying tail of the afterglow. For these reasons, in the following, we analyze a relation between the isotropic-equivalent radiated energy of the entire afterglow $E_{\text {aft }}$ and the corresponding time-integrated $v F_{v}$ spectrum peak energy $E_{\mathrm{p}}$.

\section{GRB 050315 best fit}

In Ruffini et al. (2006) we analyzed GRB 050315. Thanks to the high-quality gapless data (Vaughan et al. 2006) provided by the Swift satellite (Gehrels et al. 2004), the fit of the observed GRB 050315 light curves had to be performed simultaneously, self-consistently, and recursively in the four BAT energy bands (15-25 keV, 25-50 keV, 50-100 keV, and 100-150 keV), as well as in the XRT one $(0.2-10 \mathrm{keV})$. This fitting procedure fixed the values of $E_{\text {tot }}^{\mathrm{e}^{ \pm}}$, of $B$, and of the effective CBM distribution. It is important here to emphasize that such values are very tightly constrained by the observational data. In particular, each single value of the CBM density mask affects all the subsequent evolution of the light curves in a nonlinear way, through both the dynamical equations and the EQTSs, thus making any "piecewise" analysis of the light curves impossible. 


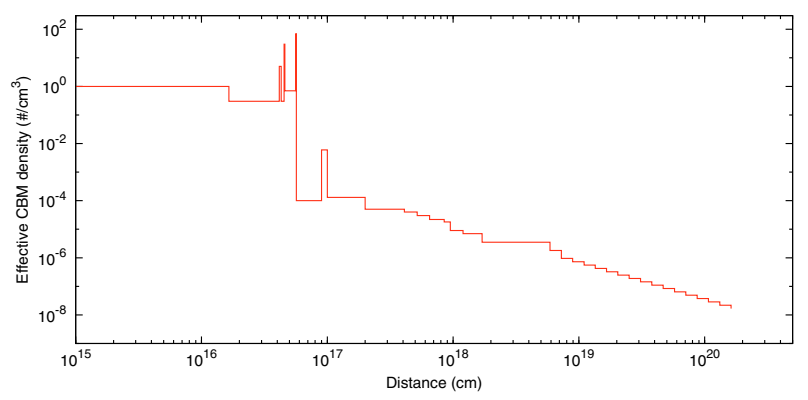

Fig. 1. The effective CBM number density inferred from the theoretical analysis of GRB 050315. Details in Ruffini et al. (2006).

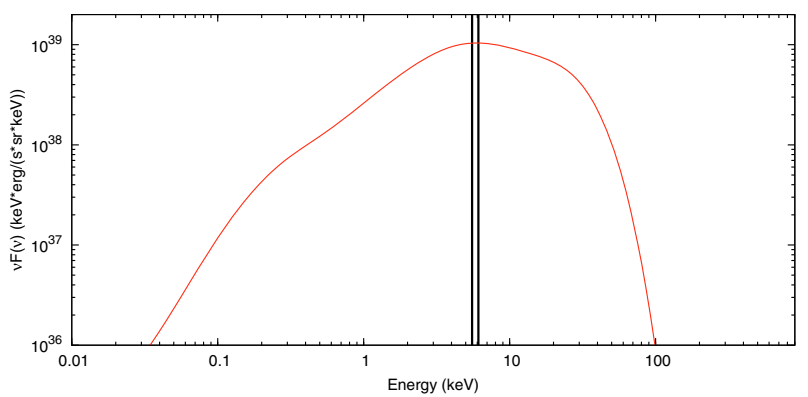

Fig. 2. The $v F_{v}$ time-integrated spectrum over the total duration of our afterglow phase for the "gedanken" GRB of the first set with total energy $E_{\text {tot }}^{e^{ \pm}}=3.40 \times 10^{51}$. The two vertical lines constrain the $5 \%$ error region around the peak. We determine $E_{\mathrm{p}}=5.82 \mathrm{keV} \pm 5 \%$.

For GRB 050315 we obtained $E_{\text {tot }}^{\mathrm{e}^{ \pm}}=1.46 \times 10^{53} \mathrm{erg}$ and $B=4.55 \times 10^{-3}$ (Ruffini et al. 2006). These two values determine the fireshell dynamics up to the transparency, and in particular they fix the ratio between $E_{\mathrm{P}-\mathrm{GRB}}$ and $E_{\mathrm{aft}}$ and the temporal separation, measured in detector arrival time, between the P-GRB and the peak of the afterglow (Ruffini et al. 2001, 2003, 2006). The effective CBM density profile inferred from our theory for GRB 050315 is shown in Fig. 1 (Ruffini et al. 2006). These values and such a profile, as stated above, were obtained when fitting the five BAT and XRT light curves of the entire GRB.

\section{The $E_{\mathrm{p}}-E_{\text {aft }}$ relation}

In our approach, only the entire afterglow emission is considered in establishing our $E_{\mathrm{p}}-E_{\text {aft }}$ relation. From this assumption one derives, in a natural way, that the Amati relation holds only for long GRBs, where the P-GRB is negligible, and not for short GRBs (Amati et al. 2007).

We can compute the "instantaneous" spectrum of GRB 050315 at each value of the detector arrival time during the entire afterglow emission. Such a spectrum sharply evolves in the arrival time, presenting a typical hard-to-soft behavior (Ruffini et al. 2006). We then computed the $v F_{v}$ time-integrated spectrum over the total duration of our afterglow phase, that is, from the end of the P-GRB up to when the fireshell reaches a Lorentz gamma factor close to unity. We can then define the energy $E_{\mathrm{p}}$ as the energy of the peak of this $v F_{v}$ time-integrated spectrum, and we look at its relation with the total energy $E_{\text {aft }}$ of the afterglow.

We construct two sets of "gedanken" GRBs at a fixed cosmological redshift, therefore independently of the cosmological model. The first set assumes the same fireshell baryon loading and effective CBM distribution as GRB 050315 (see Fig. 1) and each "gedanken" GRB differs from the others uniquely by the

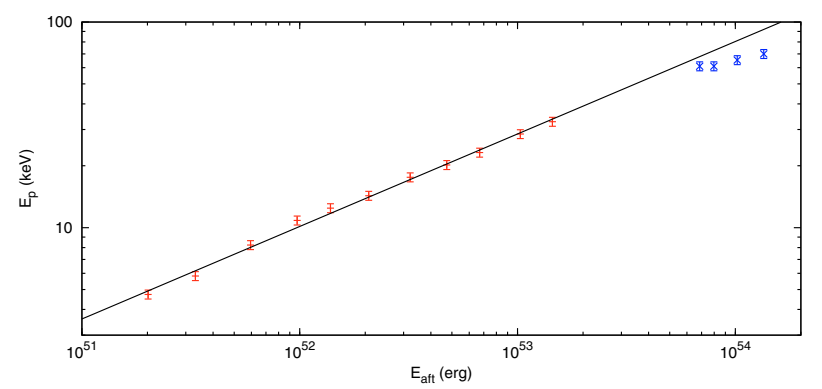

Fig. 3. The $E_{\mathrm{p}}-E_{\text {aft }}$ relation: the results of the simulations of the first set of "gedanken" GRBs (red points) are well-fitted by a power law (black line) $E_{\mathrm{p}} \propto\left(E_{\mathrm{aft}}\right)^{a}$ with $a=0.45 \pm 0.01$. The blue points are the results of the extension of the first set above $10^{53} \mathrm{erg}$.

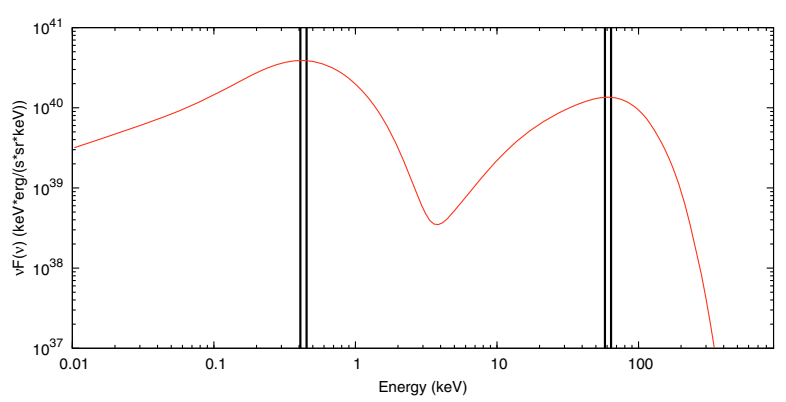

Fig. 4. The $v F_{v}$ time-integrated spectrum over the total duration of our afterglow phase for the "gedanken" GRB of the extended first set with total energy $E_{\text {tot }}^{\mathrm{e}^{ \pm}}=6.95 \times 10^{53} \mathrm{erg}$. The vertical lines constrain the $5 \%$ error region around each peak.

value of its total energy $E_{\mathrm{tot}}^{\mathrm{e}^{ \pm}}$. The second set assumes a constant effective CBM density $\sim 1$ particle $/ \mathrm{cm}^{3}$ instead of the one inferred for GRB 050315.

In our model, $E_{\mathrm{aft}}$ is a fixed value determined by $E_{\mathrm{tot}}^{\mathrm{e}^{ \pm}}$and $B$, so clearly there are no errors associated to it. Instead, $E_{\mathrm{p}}$ is evaluated from the numerically calculated spectrum, and its determination is therefore affected by the numerical resolution. Choosing a $5 \%$ error on $E_{\mathrm{p}}$, which is consistent with our numerical resolution, we checked that this value is reasonable looking at each spectrum. Figure 2 shows the time-integrated spectrum corresponding to $E_{\mathrm{tot}}^{\mathrm{e}^{ \pm}}=3.40 \times 10^{51} \mathrm{erg}$ with the error around $E_{\mathrm{p}}$.

\section{Results and discussion}

Figure 3 shows the $E_{\mathrm{p}}-E_{\text {aft }}$ relation of the "gedanken" GRBs belonging to the first set (red points). It extends over two orders of magnitude in energy, from $10^{51}$ to $10^{53} \mathrm{erg}$, and is well-fitted by a power law $E_{\mathrm{p}} \propto\left(E_{\mathrm{aft}}\right)^{a}$ with $a=0.45 \pm 0.01$. We emphasize that such a power-law slope strictly agrees with the Amati relation, namely $E_{\mathrm{p}, \mathrm{i}} \propto\left(E_{\mathrm{iso}}\right)^{a}$, with $a=0.49_{-0.05}^{+0.06}$ (Amati 2006). We recall that $E_{\mathrm{p}}$ is the observed peak energy; i.e., it is not rescaled for the cosmological redshift, because all the "gedanken" GRBs of the set are at the same redshift of GRB 050315, namely $z=1.949$ (Vaughan et al. 2006). The normalization is clearly different from the Amati one.

If we try to extend the first sample of "gedanken" GRBs below $10^{51} \mathrm{erg}$, the relevant CBM distribution would be for $r \lesssim 10^{16} \mathrm{~cm}$, where no data are available from the GRB 050315 observations. If we try to extend the first set of "gedanken" GRBs above $10^{53} \mathrm{erg}$, we notice that for $E_{\mathrm{tot}}^{\mathrm{e}^{ \pm}} \gtrsim 10^{54} \mathrm{erg}$ the small "bump", which can be noticed between 0.2 and $1.0 \mathrm{keV}$ in the spectrum of Fig. 2, evolves into a low-energy second spectral peak that is even higher than the high-energy one (see Fig. 4). 


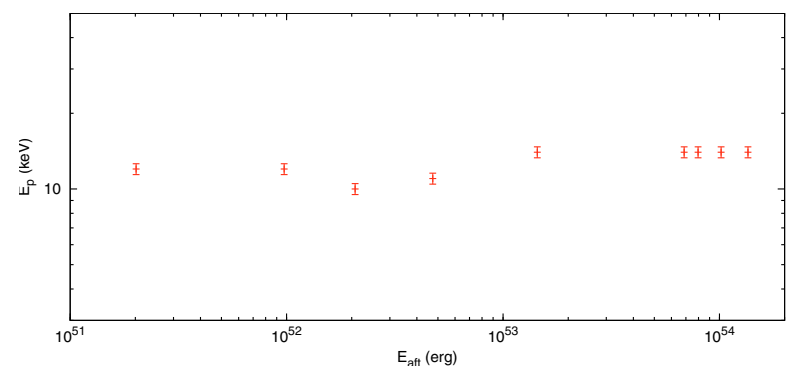

Fig. 5. The second set of "gedanken" GRBs. Clearly, in this case there in no relation between $E_{\mathrm{p}}$ and $E_{\mathrm{aft}}$.

We are currently investigating whether this low-energy second peak is a real, theoretically predicted spectral feature that may be observed in the future in highly energetic sources. There is also the other possibility that the low-energy and late part of our GRB 050315 fit is not enough constrained by the XRT observational data so that this effect is magnified by the $E_{\text {tot }}^{\mathrm{e}^{ \pm}}$rescaling.

The high-energy spectral peak is due to the emission at the peak of the afterglow, and therefore due to the so-called "prompt emission". The low-energy one is due to late-time soft X-ray emission. Therefore, the high-energy spectral peak is the relevant one for the Amati relation. We find indeed that such a high-energy spectral peak still fulfills the $E_{\mathrm{p}}-E_{\text {aft }}$ relation for $E_{\mathrm{tot}}^{\mathrm{e}^{ \pm}} \sim 10^{54} \mathrm{erg}$, with a possible saturation for $E_{\mathrm{tot}}^{\mathrm{e}^{ \pm}}>10^{54} \mathrm{erg}$ (see Fig. 3).

Figure 5 clearly shows that in the second set of "gedanken" GRBs, built assuming a constant effective CBM density $\sim 1$ particle $/ \mathrm{cm}^{3}$, instead of the one specifically inferred for GRB 050315 , there in no relation between $E_{\mathrm{p}}$ and $E_{\mathrm{aft}}$.

\section{Conclusions}

The high-quality Swift data, for the first time giving gapless and multiwavelength coverage from the GRB trigger all the way to the latest afterglow phases, have led to a complete fit of the GRB 050315 multiband light curves based on our fireshell model. We fixed the free parameters describing the source and determined the instantaneous and time-integrated spectra during the entire afterglow.

Starting from this, we examined two sets of "gedanken" GRBs, constructed at a fixed cosmological redshift. The first set assumes the same fireshell baryon loading and effective CBM distribution as GRB 050315, and each "gedanken" GRB differs from the others uniquely by the value of its total energy $E_{\text {tot }}^{\mathrm{e}^{ \pm}}$. The second set assumes a constant effective CBM density $\sim 1$ particle $/ \mathrm{cm}^{3}$ instead of the one inferred for GRB 050315 .

Recalling that the "canonical" GRB light curve in the fireshell model is composed of two well-separated components, the P-GRB, and the entire afterglow, we looked for a relation in both sets between the isotropic-equivalent radiated energy of the entire afterglow $E_{\text {aft }}$ and the corresponding time-integrated $v F_{v}$ spectrum peak energy $E_{\mathrm{p}}: E_{\mathrm{p}} \propto\left(E_{\mathrm{aft}}\right)^{a}$. In doing so, we assumed that the Amati relation is directly linked to the interaction between the accelerated baryons and the CBM. The P-GRBs, which originate from the fireshell transparency, do not fulfill the Amati relation in our approach. Consequently, the short GRBs, which have a vanishing afterglow with respect to the P-GRB, should also not fulfill the Amati relation. This last point is supported by the observational evidence (Amati 2006).
We notice that the first set of "gedanken" GRBs fulfills the $E_{\mathrm{p}} \propto\left(E_{\mathrm{aft}}\right)^{a}$ relation very well with $a=0.45 \pm 0.01$. This slope strongly agrees with the Amati relation. In contrast, no relation between $E_{\mathrm{p}}$ and $E_{\mathrm{aft}}$ seems to hold for the second set. We conclude that the Amati relation originates from the detailed structure of the effective CBM.

Turning now to the analogies and differences between our $E_{\mathrm{p}}-E_{\text {aft }}$ relation and the Amati one, our analysis excludes the P-GRB from the prompt emission, extends all the way to the latest afterglow phases, and is independent of the assumed cosmological model, since all "gedanken" GRBs are at the same redshift. The Amati relation, on the other hand, includes the PGRB, focuses only on the prompt emission, being therefore influenced by the instrumental threshold that fixes the end of the prompt emission, and depends on the assumed cosmology. This might explain the intrinsic scatter observed in the Amati relation (Amati 2006). Our theoretical work is a first unavoidable step toward supporting the use of the empirical Amati relation for measuring the cosmological parameters.

Acknowledgements. We thank the anonymous referee for his/her very constructive advice.

\section{References}

Amati, L. 2006, MNRAS, 372, 233

Amati, L., Frontera, F., Tavani, M., et al. 2002, A\&A, 390, 81

Amati, L., Della Valle, M., Frontera, F., et al. 2007, A\&A, 463, 913

Amati, L., Guidorzi, C., Frontera, F., et al. 2008 [arXiv:0805.0377]

Bernardini, M. G., Bianco, C. L., Chardonnet, P., et al. 2005, ApJ, 634, L29

Bernardini, M. G., Bianco, C. L., Caito, L., et al. 2007, A\&A, 474, L13

Bernardini, M. G., Bianco, C. L., Caito, L., et al. 2008, in Relativistic Astrophysics, ed. C. L. Bianco, \& S. S. Xue, AIP Conf. Ser., 966, 7

Bianco, C. L., \& Ruffini, R. 2004, ApJ, 605, L1

Bianco, C. L., \& Ruffini, R. 2005, ApJ, 620, L23

Bianco, C. L., Ruffini, R., \& Xue, S.-S. 2001, A\&A, 368, 377

Bianco, C. L., Bernardini, M. G., Caito, L., et al. 2008, in Relativistic Astrophysics, ed. C. L. Bianco, \& S. S. Xue, AIP Conf. Ser., 966, 12

Caito, L., Bernardini, M. G., Bianco, C. L., et al. 2008, in Relativistic Astrophysics, ed. C. L. Bianco, \& S. S. Xue, AIP Conf. Ser., 966, 16 Dainotti, M. G., Bernardini, M. G., Bianco, C. L., et al. 2007, A\&A, 471, L29 Firmani, C., Ghisellini, G., Avila-Reese, V., \& Ghirlanda, G. 2006, MNRAS, 370,185

Gehrels, N., Chincarini, G., Giommi, P., et al. 2004, ApJ, 611, 1005

Ghirlanda, G., Ghisellini, G., \& Lazzati, D. 2004, ApJ, 616, 331

Leibundgut, B. 2001, ARA\&A, 39, 67

Liang, E., \& Zhang, B. 2005, ApJ, 633, 611

Norris, J. P., \& Bonnell, J. T. 2006, ApJ, 643, 266

Riess, A. G., Nugent, P. E., Gilliland, R. L., et al. 2001, ApJ, 560, 49

Ruffini, R., Salmonson, J. D., Wilson, J. R., \& Xue, S.-S. 2000, A\&A, 359, 855

Ruffini, R., Bianco, C. L., Chardonnet, P., Fraschetti, F., \& Xue, S.-S. 2001, ApJ, 555, L113

Ruffini, R., Bianco, C. L., Chardonnet, P., Fraschetti, F., \& Xue, S.-S. 2002, ApJ, 581, L19

Ruffini, R., Bianco, C. L., Chardonnet, P., et al. 2003, in Cosmology and Gravitation, ed. M. Novello, \& S. E. Perez Bergliaffa, AIP Conf. Ser., 668, 16 Ruffini, R., Bianco, C. L., Chardonnet, P., et al. 2004, IJMPD, 13, 843 Ruffini, R., Bianco, C. L., Chardonnet, P., et al. 2005a, IJMPD, 14, 97 Ruffini, R., Fraschetti, F., Vitagliano, L., \& Xue, S.-S. 2005b, IJMPD, 14, 131 Ruffini, R., Bernardini, M. G., Bianco, C. L., et al. 2006, ApJ, 645, L109 Ruffini, R., Bernardini, M. G., Bianco, C. L., et al. 2007, in XIIth Brazilian School of Cosmology and Gravitation, ed. M. Novello, \& S. E. Perez Bergliaffa, AIP Conf. Ser., 910, 55

Sakamoto, T., Barbier, L., Barthelmy, S. D., et al. 2006, ApJ, 636, L73 Tagliaferri, G., Antonelli, L. A., Chincarini, G., et al. 2005, A\&A, 443, L1 Vaughan, S., Goad, M. R., Beardmore, A. P., et al. 2006, ApJ, 638, 920 Yonetoku, D., Murakami, T., Nakamura, T., et al. 2004, ApJ, 609, 935 